\title{
Modeling of Guided-wave Excitation by Finite-dimensional Piezoelectric Transducers in Composite Plates
}

\author{
Ajay Raghavan $^{1}$ and Carlos E.S. Cesnik ${ }^{2}$ \\ Department of Aerospace Engineering, University of Michigan, 1320 Beal Ave., Ann Arbor, Michigan 48109-2140
}

\begin{abstract}
This paper addresses modeling of guided-wave excitation by surface-bonded piezoelectric wafer transducers in multi-layered composite plate structures. Each of the individual layers is assumed to have unidirectional fibers in an epoxy matrix and is modeled as being transversely isotropic. The piezoelectric actuators are modeled as causing shear traction along their edges on the plate surface and uncoupled actuator-substrate dynamics are assumed. The formulation is generic enough to accommodate arbitrary shape actuators, and specific expressions are derived for the cases of rectangular and ring-shapes. The surface and interfacial conditions are implemented using the global matrix approach. Since the three-dimensional guided-wave field is modeled without using reduced structural formulations, all possible guided-wave modes are captured. The two-dimensional spatial Fourier transform is used to solve the problem, and a rigorous inversion procedure is outlined. These models are implemented numerically and results in the form of harmonic radiation plots are presented for various configurations.
\end{abstract}

\section{Introduction}

$\mathrm{T}$ he prospect of having aerospace and other structures instrumented with on-board damage prognosis systems has generated a lot of interest in the area of structural health monitoring (SHM) over the past decade. The hope is that such systems would be able to regularly scan the structure for damage and warn the user in near-real time about any incipient damage. They should also be able to tie in with prognostic algorithms to furnish estimates about the remaining service life of the structure. The presence of damage prognosis systems may increase safety. In addition, they could enable a transition from schedule-driven inspection to condition-based maintenance. The monetary and labor savings benefits of such systems may be also very significant.

Another growing trend in aerospace structures is the widespread use of composites. The primary advantage of using composites is their higher stiffness-to-mass ratio compared to metals, which translates into significant fuel and operational-cost savings. In addition, they have better corrosion resistance and can be tailored for preferentially bearing loads along specific directions. However, they are more susceptible to impact damage. Impact can cause damage in the form of delaminations or cracks, reduce load-bearing capability, and potentially lead to structural failure. The capability of health monitoring could increase confidence in the use of composite structures by alerting operators about damage from unexpected impact events.

A. Guided-wave structural health monitoring

While several approaches have been examined for SHM, guided-wave (GW) methods have shown potential to actively interrogate large structural areas with a sparse network of transducers. These essentially involve exciting the structure with high frequency stress waves and processing the difference in structural response with respect to a baseline signal for the pristine condition, from which damage, if present, can be detected and characterized. A detailed survey on GW SHM, including fundamentals and early history, is presented in a review paper by the authors ${ }^{1}$. GWs can be defined as stress waves forced to follow a path defined by the material boundaries of the structure. Aerospace vehicles usually consist of different substructures, each of which can act as waveguides, thereby making them attractive application areas for GW SHM. In SHM, typically surface-bonded piezoelectric wafer transducers (called "piezos" in this work) are used.

\footnotetext{
${ }^{1}$ Graduate Research Assistant and AIAA Member

2 Associate Professor and Associate Fellow, AIAA; Corresponding Author; Email: cesnik@umich.edu; Phone: 1734-764-3397; Fax: 1-734-763-0578
} 
However, GW SHM has some caveats associated with it. In isotropic structures, typically, more than one mode is possible in a waveguide at any frequency. Furthermore, as shown in Figure 1(a), each mode has a unique dispersion curve, which represents the relation between phase velocity and frequency. In composite structures, this is further complicated by the directional dependence of wavespeeds, due to the difference in elastic properties along different directions (e.g., see Figure 1 (b)). Therefore, it is important to have a fundamental understanding of GW propagation and characterize the GW field excited and sensed by the transducers being used. Earlier work by the authors $^{2,3}$ has addressed this issue for isotropic structures. With composite materials becoming increasingly common in aerospace structures, there is a need to address that in composite plate structures as well. That is the aim of the present paper.

\section{B. Previous work}

The theory of free GW propagation in isotropic, anisotropic and layered materials for various geometries as well as excitation using conventional NDE ultrasonic transducers is well-documented ${ }^{4}$. The free GW modes in isotropic plates and shells were first studied by Lamb ${ }^{5}$ and Gazis ${ }^{6}$ respectively using the theory of elasticity. Earlier works on modeling excitation of GW fields using the theory of elasticity mostly used two-dimensional (2-D) models, wherein variations along one direction in the plane of the plate were ignored. Work done on modeling excitation in isotropic structures has been reviewed in the authors' survey paper ${ }^{1}$. Some works have examined GW excitation by transducers for non-destructive testing (NDT) in composites. Ditri and Rose ${ }^{7}$ used 2-D elasticity models along with the normal modes expansion technique to describe GW excitation in composites. $\mathrm{Mal}^{8}$ and Lih and $\mathrm{Mal}^{9}$ developed a theoretical formulation to solve for the problem of forced GW excitation by finite-dimensional sources using 3-D elasticity in multilayered composite plates. The Fourier spatial integrals were inverted using a numerical scheme. Viscoelastic damping was addressed, and specifically, the cases of excitation by NDT transducers and acoustic emission were solved based on the developed formulation. More recently, Mal and Banerjee ${ }^{10}$ proposed a semianalytical approach for the inversion of the spatial domain Fourier transform, using the residue theorem along one direction in the plane of the plate and then numerically evaluating the integral along the other. However, that approach leads to a standing wave in the first direction, which is counter-intuitive.

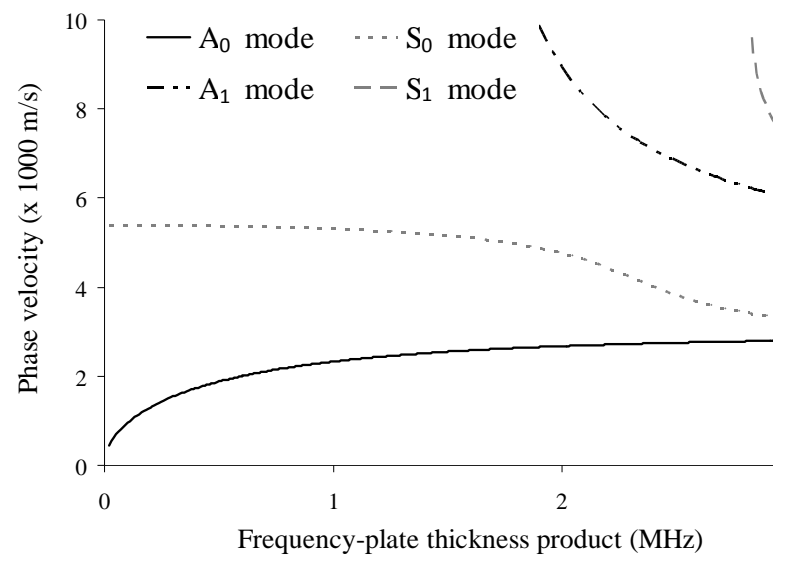

(a)

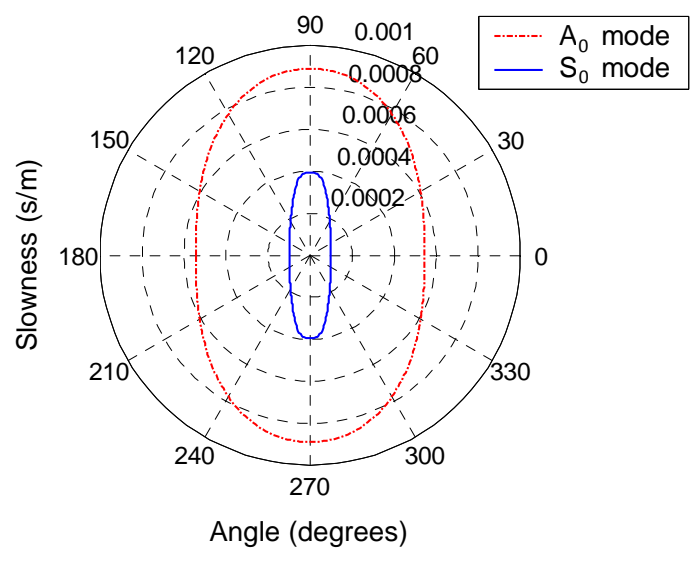

(b)

Figure 1. (a) Dispersion curve (plot of phase velocity versus frequency) for the first four Lamb modes in an aluminum alloy plate (isotropic) and (b) Slowness surfaces (plot of inverse of phase velocity versus direction) in a 1-mm thick unidirectional graphite-epoxy composite plate at $500 \mathrm{kHz}$ (with graphite fibers along $\mathbf{0}^{\circ} / \mathbf{1 8 0}^{\circ}$ ).

In comparison, less modeling work has been done for GW testing using structurally integrated piezos for SHM. Due to this, often little or no theoretical basis is provided by researchers for their choice of the various testing parameters involved such as transducer geometry, dimensions, location and materials, excitation frequency, bandwidth among others. Among the works that have sought to bridge this gap, Moulin et al. ${ }^{11}$ used a coupled finite element-normal modes expansion method to model GW excitation in composite plates with piezos. This was also a 2-D analysis. Some researchers (Lin and Yuan ${ }^{12}$, Rose and Wang $^{13}$, Veidt et al. ${ }^{14}$ ) have looked at using Mindlin plate theory for modeling GW excitation by circular and/or rectangular piezos in isotropic plates. That approach yields approximate solutions and cannot model higher GW modes. Giurgiutiu ${ }^{15}$ studied the 2-D harmonic excitation of Lamb-waves in isotropic plates by infinitely wide surface-bonded piezos. As he suggested, the key difference 
between NDE transducers and surface-bonded piezos is that the former operate by "tapping" or causing normal traction on the surface, while the latter operate by "pinching" or causing shear traction at the actuator edges on the structural surface. Thus, the problem of modeling the 3-D GW field excited by finite dimensional piezos in composite plates has not received much attention.

C. Objective of this work

In previous work by the authors ${ }^{2}$, analytical solutions were presented for the problem of GW excitation and sensing by finite-dimensional piezo transducers in isotropic plates using 3-D elasticity. The actuators were modeled as causing surface shear traction along their free edges, while the sensors were modeled as sensing the average in-plane extensional strain over their surface area. Those were validated with numerical and experimental results. In the present paper, multilayered composite plates are addressed. The transducer models are identical to that in the authors' earlier work ${ }^{2}$. The formulation and notation for the underlying multilayered composite plate is largely adapted from Lih and $\mathrm{Mal}^{9}$ and are summarized here for completeness. A semi-analytical approach is used for the spatial Fourier inversion, which is distinct from that in Ref. 10. The new approach in the present work yields traveling waves along both directions in the plane of the plate, as one would expect.

\section{Theoretical Formulation}

In this section, a general expression for the GW field excited by an arbitrary shape (finite dimensional) piezoactuator surface-bonded on a multilayered composite plate is derived. Consider an infinite $N$-layered composite plate of total thickness $H$, with such an actuator bonded on one free surface, as illustrated in Figure 2. The origin is located on the free surface with the actuator and the $X_{3}$-axis is normal to the plate surface. The individual layers are assumed to have unidirectional fibers in a matrix and are modeled as being transversely isotropic with uniform density. This is a reasonable assumption if the GW wavelength is large compared to the inter-fiber spacing and the fiber diameter. The solution procedure consists of the following four components (illustrated in Figure 3):

(a) First, one sets up the 3-D governing equations of motion for the bulk composite medium. The 2-D Fourier transform is applied (or equivalently, plane waves propagating at a given angle in the plane of the fibers are assumed). This yields the free-wave solution in terms of the eigenvectors and possible wavenumbers through the thickness of the fibers.

(b) Then, one imposes the free-surface conditions of the plate along with the continuity conditions across interfaces (using the global matrix formulation). This also gives the allowable in-plane wavenumbers for the possible GW modes.

(c) Next, the forcing function due to the presence of the surface-bonded piezo-actuators is imposed (assuming they cause shear traction along their free edges). This gives the solution in terms of a 2-D Fourier integral in the wavenumber domain.

(d) Finally, the 2-D wavenumber-domain Fourier integral is inverted (semi-analytically) to yield the GW field due to harmonic excitation by the piezo-actuator. The response to an arbitrary excitation waveform can then be obtained by integrating the individual harmonic components of the time-domain signal (i.e., inverting the frequency-domain Fourier integral).

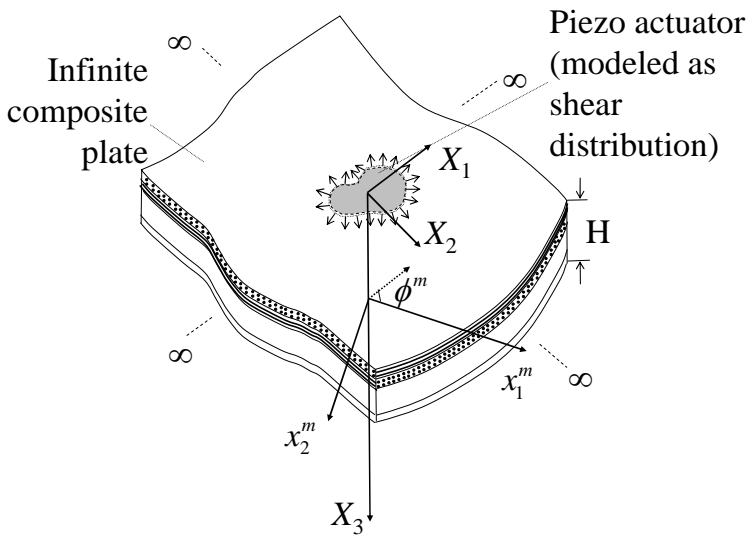

Figure 2. Infinite multilayered composite plate with arbitrary shape surface-bonded piezo actuator and piezo sensor 




(a) Plane wave solution for infinite bulk composite (Ref. 16)

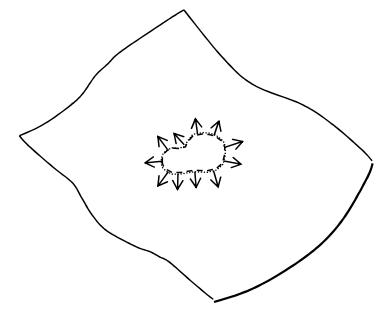

(c) Forcing function due to surface-bonded piezo (shear traction along edge)



(b) Free GW solution for infinite multilayered composite plate (Ref. 9)

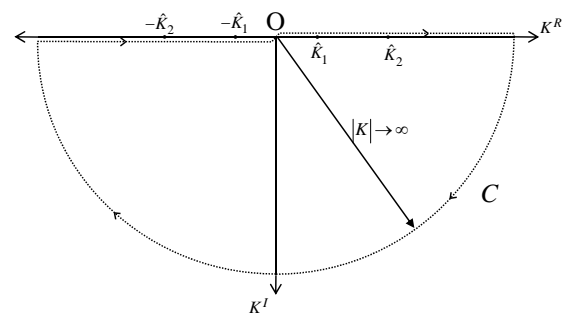

(d) Wavenumber Fourier integral inversion (residue calculus)

Figure 3. Illustration of solution procedure

Among these, parts (a) and (b) are adopted from Refs. 9 and 16. Part (c) is based on the authors' earlier work for isotropic plates ${ }^{2,3}$. The details of the solution procedure are explained in the following sub-sections.

A. Bulk waves in fiber-reinforced composites

First, consider the general solution for bulk waves in a transversely isotropic medium. The equations of motion for the bulk medium in each layer are:

$$
\nabla c \nabla^{T} \overline{\boldsymbol{u}}=\rho \ddot{\overline{\boldsymbol{u}}}
$$

where $\overline{\boldsymbol{u}}$ is the displacement vector, $\boldsymbol{c}$ is the stiffness matrix, the $\cdot$ over a variable indicates derivative with respect to time, $\rho$ is the material density, and the operator $\nabla$ is defined as:

$$
\nabla=\left[\begin{array}{cccccc}
\frac{\partial}{\partial x_{1}} & 0 & 0 & 0 & \frac{\partial}{\partial x_{3}} & \frac{\partial}{\partial x_{2}} \\
0 & \frac{\partial}{\partial x_{2}} & 0 & \frac{\partial}{\partial x_{3}} & 0 & \frac{\partial}{\partial x_{1}} \\
0 & 0 & \frac{\partial}{\partial x_{3}} & \frac{\partial}{\partial x_{2}} & \frac{\partial}{\partial x_{1}} & 0
\end{array}\right]
$$

If the fibers are oriented along the 1-direction in the local coordinate system $\left(x_{1}, x_{2}, x_{3}\right)$ of the material, the stressstrain relation and the stiffness matrix $\boldsymbol{c}$ for a transversely isotropic material are:

$$
\left[\begin{array}{c}
\sigma_{11} \\
\sigma_{22} \\
\sigma_{33} \\
\sigma_{23} \\
\sigma_{31} \\
\sigma_{12}
\end{array}\right]=\boldsymbol{c}\left[\begin{array}{c}
u_{1,1} \\
u_{2,2} \\
u_{3,3} \\
u_{2,3}+u_{3,2} \\
u_{1,3}+u_{3,1} \\
u_{2,1}+u_{1,2}
\end{array}\right] \quad ; \quad \boldsymbol{c}=\left[\begin{array}{cccccc}
c_{11} & c_{12} & c_{12} & 0 & 0 & 0 \\
c_{12} & c_{22} & c_{23} & 0 & 0 & 0 \\
c_{12} & c_{23} & c_{22} & 0 & 0 & 0 \\
0 & 0 & 0 & c_{44} & 0 & 0 \\
0 & 0 & 0 & 0 & c_{55} & 0 \\
0 & 0 & 0 & 0 & 0 & c_{55}
\end{array}\right] \text {, with } c_{44}=\frac{c_{22}-c_{23}}{2}
$$

Here $\sigma_{i j}$, with $i$ and $j$ taking integer values from 1 to 3 , are the stress components. Next, constants are introduced that correspond to the squares of bulk wave speeds along different directions: 


$$
\begin{aligned}
& a_{1}=c_{22} / \rho \text { (dilatational wave normal to the fiber direction) } \\
& a_{2}=c_{11} / \rho \text { (dilatational wave along the fiber direction) } \\
& a_{3}=\left(c_{12}+c_{55}\right) / \rho \text { (shear wave in the plane of isotropy) } \\
& a_{4}=\left(c_{22}-c_{23}\right) / 2 \rho=c_{44} / \rho \text { (shear wave along the fiber direction) } \\
& a_{5}=c_{55} / \rho \text { (shear wave in the plane of isotropy) }
\end{aligned}
$$

Viscoelastic damping can be modeled by the use of complex stiffness constants. Suppose the wavenumber components are $\xi_{1}, \xi_{2}$ and $\zeta$ along the 1-, 2- and 3- local directions, respectively. Furthermore, without loss of generality, consider harmonic excitation at angular frequency $\omega$. Then the wave field is of the form:

$$
\overline{\mathbf{u}}=\boldsymbol{C} e^{-i\left(\xi_{1} x_{1}+\xi_{2} x_{2}+\zeta x_{3}-\omega t\right)}
$$

where $\boldsymbol{C}$ is a vector of constants. Then, from Eqs. (1)-(5), one obtains the Christoffel equation:

$$
\left[\begin{array}{ccc}
c_{11} \xi_{1}^{2}+c_{55}\left(\xi_{2}^{2}+\zeta^{2}\right) & \left(c_{12}+c_{55}\right) \xi_{1} \xi_{2} & \left(c_{12}+c_{55}\right) \xi_{1} \zeta \\
\left(c_{12}+c_{55}\right) \xi_{1} \xi_{2} & c_{55} \xi_{1}^{2}+c_{22} \xi_{2}^{2}+c_{44} \zeta^{2} & \left(c_{23}+c_{44} \xi_{2} \zeta\right. \\
\left(c_{12}+c_{55}\right) \xi_{1} \zeta & \left(c_{23}+c_{44}\right) \xi_{2} \zeta & c_{55} \xi_{1}^{2}+c_{44} \xi_{2}^{2}+c_{22} \zeta^{2}
\end{array}\right]\left[\begin{array}{l}
\bar{u}_{1} \\
\bar{u}_{2} \\
\bar{u}_{3}
\end{array}\right]=\rho \omega^{2}\left[\begin{array}{c}
\bar{u}_{1} \\
\bar{u}_{2} \\
\bar{u}_{3}
\end{array}\right]
$$

For fixed values of $\xi_{1}, \xi_{2}$, and $\omega$, there are six possible roots $\pm \zeta_{i}, i=1$ to 3 , of this equation. The first two pairs of roots correspond to pairs of quasi-longitudinal waves and quasi-shear waves ${ }^{9,16}$. The wavenumbers in the thickness direction for these four roots are, respectively:

$$
\begin{aligned}
& \zeta_{1}^{2}=-\xi_{2}^{2}+b_{1} \quad ; \quad \zeta_{2}^{2}=-\xi_{2}^{2}+b_{2} \\
& b_{1}=-\left(\frac{\beta}{2 \alpha}\right)-\sqrt{\left(\frac{\beta}{2 \alpha}\right)^{2}-\frac{\gamma}{\alpha}} ; \quad b_{2}=-\left(\frac{\beta}{2 \alpha}\right)+\sqrt{\left(\frac{\beta}{2 \alpha}\right)^{2}-\frac{\gamma}{\alpha}} \\
& \alpha=a_{1} a_{5} ; \beta=\left(a_{1} a_{2}+a_{5}^{2}-a_{3}^{2}\right) \xi_{1}^{2}-\omega^{2}\left(a_{1}+a_{5}\right) \quad ; \quad \gamma=\left(a_{2} \xi_{1}^{2}-\omega^{2}\right)\left(a_{5} \xi_{1}^{2}-\omega^{2}\right)
\end{aligned}
$$

The third pair of roots corresponds to pure shear waves and their through-thickness wavenumbers are given by:

$$
\zeta_{3}^{2}=-\xi_{2}^{2}+\left(\omega^{2}-a_{5} \xi_{1}^{2}\right) / a_{4}
$$

The displacement eigenvectors resulting from Eq. (6) corresponding to these roots are:

where

$$
\begin{aligned}
& \boldsymbol{e}_{1}=\left[\begin{array}{lll}
i \xi_{1} q_{11} & i \xi_{2} q_{21} & i \zeta_{1} q_{21}
\end{array}\right]^{T} \\
& \boldsymbol{e}_{2}=\left[\begin{array}{lll}
i \xi_{1} q_{12} & i \xi_{2} q_{22} & i \zeta_{2} q_{22}
\end{array}\right]^{T} \\
& \boldsymbol{e}_{3}=\left[\begin{array}{lll}
0 & i \zeta_{3} & -i \xi_{2}
\end{array}\right]^{T}
\end{aligned}
$$

$$
\begin{array}{ll}
q_{11}=a_{3} b_{1} ; & q_{21}=\omega^{2}-a_{2} \xi_{1}^{2}-a_{5} b_{1} \\
q_{21}=a_{3} b_{2} ; & q_{22}=\omega^{2}-a_{2} \xi_{1}^{2}-a_{5} b_{2}
\end{array}
$$

and the other three eigenvectors $\boldsymbol{e}_{4}, \boldsymbol{e}_{5}$ and $\boldsymbol{e}_{6}$ are obtained by replacing $\zeta_{i}$ by $-\zeta_{i}$. The general solution for the displacement vector is then given by:

$$
\overline{\boldsymbol{u}}=\left(C_{1+} \boldsymbol{e}_{1} e^{i \zeta_{1} x_{3}}+C_{2+} \boldsymbol{e}_{2} e^{i \zeta_{2} x_{3}}+C_{3+} \boldsymbol{e}_{3} e^{i \zeta_{3} x_{3}}+C_{1-} \boldsymbol{e}_{4} e^{-i \zeta_{1} x_{3}}+C_{2-} \boldsymbol{e}_{5} e^{-i \zeta_{2} x_{3}}+C_{3-} \boldsymbol{e}_{6} e^{-i \zeta_{3} x_{3}}\right) e^{-i\left(\xi_{1} x_{1}+\xi_{2} x_{2}-\omega t\right)}
$$

where $C_{i \pm}, i=1$ to 3 , are free constants.

\section{B. Assembling the laminate global matrix from the individual layer matrices}

With the general solution for the bulk medium in place, one can then seek the particular solution for the problem at hand. As mentioned earlier, the equations in this particular sub-section are from Lih and $\mathrm{Mal}^{9}$, and details can be found there. They are only summarized here.

Due to the different orientations of the fibers in the different layers, it is useful to work with a global coordinate system $\left(X_{1}, X_{2}, X_{3}\right)$ distinct from the local coordinate system, for which the $x_{1}^{m}$-axis is aligned with the fiber direction. However, the $X_{3}$ - and $x_{3}^{m}$-axes are coincident and the two coordinate systems differ only in the plane of the plate. One can relate the displacement vector $\boldsymbol{u}$ in the global system and $\overline{\boldsymbol{u}}$ in the local system using the transformation matrix $\boldsymbol{L}^{m}$ (with the superscript $m$ indicating the layer number between 1 and $N$, and $\phi^{m}$ being the angle between the $X_{1}$ - and $x_{1}^{m}$-axes): 


$$
\boldsymbol{u}=\boldsymbol{L}^{m} \overline{\boldsymbol{u}} \quad \text { where } \boldsymbol{L}^{m}=\left[\begin{array}{ccc}
\cos \phi^{m} & -\sin \phi^{m} & 0 \\
\sin \phi^{m} & \cos \phi^{m} & 0 \\
0 & 0 & 1
\end{array}\right]
$$

The surface traction conditions for this problem are:

$$
\begin{aligned}
\sigma_{i 3}^{1}\left(X_{1}, X_{2}, 0\right) & =f_{i}\left(X_{1}, X_{2}, 0\right) \\
\sigma_{i 3}^{N}\left(X_{1}, X_{2}, H\right) & =0 ; i=1,2,3
\end{aligned}
$$

where the functions $f_{1}$ and $f_{2}$ depend on the shape of the actuator and $f_{3}=0$. In addition, traction and displacement continuity must be maintained across the interfaces between the different layers. The 2-D spatial Fourier transform is used to ease solution of this problem. For a generic variable $\psi$, it is defined by:

$$
\Psi\left(K_{1}, K_{2}\right)=\int_{-\infty}^{\infty} \int_{-\infty}^{\infty} \psi\left(X_{1}, X_{2}\right) e^{i\left(K_{1} X_{1}+K_{2} X_{2}\right)} d X_{1} d X_{2}
$$

and the inverse transform is given by:

$$
\psi\left(X_{1}, X_{2}\right)=\frac{1}{4 \pi^{2}} \int_{-\infty}^{\infty} \int_{-\infty}^{\infty} \Psi\left(K_{1}, K_{2}\right) e^{-i\left(K_{1} X_{1}+K_{2} X_{2}\right)} d K_{1} d K_{2}
$$

Let $\boldsymbol{U}, \Sigma$ and $\boldsymbol{F}$ denote the 2-D spatial Fourier transform of the variables $\boldsymbol{u}, \boldsymbol{\sigma}$ and $\boldsymbol{f}$, respectively. Furthermore, as for the bulk medium solution, without loss of generality, harmonic excitation at angular frequency $\omega$ is considered (the $e^{i \omega t}$ factor is suppressed for convenience and is brought back at the end). Since continuity of both traction and displacement has to be ensured across all interfaces, it is convenient to work with a "displacement-stress vector" $\boldsymbol{S}^{\boldsymbol{m}}$ in the transformed domain defined as:

$$
\boldsymbol{S}^{\boldsymbol{m}}\left(X_{3}\right)=\left\{\boldsymbol{U}^{\boldsymbol{m}}\left(X_{3}\right) \sum_{i 3}^{m}\left(X_{3}\right)\right\}^{T}
$$

Then, from Eqs. (3), (11), and (12):

$$
\boldsymbol{S}^{m}\left(X_{3}\right)=\left[\begin{array}{cc}
\boldsymbol{L}^{m} & \mathbf{0} \\
\mathbf{0} & \boldsymbol{L}^{m}
\end{array}\right]\left[\begin{array}{ll}
\boldsymbol{Q}_{11}^{m} & \boldsymbol{Q}_{12}^{m} \\
\boldsymbol{Q}_{21}^{m} & \boldsymbol{Q}_{22}^{m}
\end{array}\right]\left[\begin{array}{cc}
\boldsymbol{E}_{+}^{m}\left(X_{3}\right) & \mathbf{0} \\
\mathbf{0} & \boldsymbol{E}_{-}^{m}\left(X_{3}\right)
\end{array}\right]\left\{\begin{array}{l}
\boldsymbol{C}_{+}^{m} \\
\boldsymbol{C}_{-}^{m}
\end{array}\right\} \equiv \boldsymbol{Q}^{m}\left(X_{3}\right) \boldsymbol{C}^{m}
$$

where:

$$
\begin{aligned}
& \boldsymbol{Q}_{11}^{m}=\left[\begin{array}{lll}
\boldsymbol{e}_{1}^{m} & \boldsymbol{e}_{2}^{m} & \boldsymbol{e}_{3}^{m}
\end{array}\right] \quad ; \quad \boldsymbol{Q}_{12}^{m}=\left[\begin{array}{lll}
\boldsymbol{e}_{4}^{m} & \boldsymbol{e}_{5}^{m} & \boldsymbol{e}_{6}^{m}
\end{array}\right] \\
& \boldsymbol{Q}_{21}^{m}=\left[\begin{array}{ccc}
-\rho a_{5} \xi_{1} \zeta_{1}\left(q_{11}+q_{21}\right) & -\rho a_{5} \xi_{1} \zeta_{2}\left(q_{11}+q_{22}\right) & \rho a_{5} \xi_{1} \xi_{2} \\
-2 \rho a_{4} \xi_{2} \zeta_{1} q_{21} & -2 \rho a_{4} \xi_{2} \zeta_{2} q_{22} & \rho a_{4}\left(\xi_{2}^{2}-\zeta_{3}^{2}\right) \\
\mu_{1} & \mu_{2} & 2 \rho a_{4} \xi_{2} \zeta_{3}
\end{array}\right] \\
& \boldsymbol{Q}_{22}^{m}=\left[\begin{array}{ccc}
\rho a_{5} \xi_{1} \zeta_{1}\left(q_{11}+q_{21}\right) & \rho a_{5} \xi_{1} \zeta_{2}\left(q_{11}+q_{22}\right) & \rho a_{5} \xi_{1} \xi_{2} \\
2 \rho a_{4} \xi_{2} \zeta_{1} q_{21} & 2 \rho a_{4} \xi_{2} \zeta_{2} q_{22} & \rho a_{4}\left(\xi_{2}^{2}-\zeta_{3}^{2}\right) \\
\mu_{1} & \mu_{2} & -2 \rho a_{4} \xi_{2} \zeta_{3}
\end{array}\right] \\
& \boldsymbol{C}^{m}=\left[\begin{array}{ll}
\boldsymbol{C}_{+}^{m} & \boldsymbol{C}_{-}^{m}
\end{array}\right]^{T} \\
& \mu_{1}=\rho\left[\left(a_{5}-a_{3}\right) \xi_{1}^{2} q_{11}-\left(a_{1}-2 a_{4}\right) \xi_{2}^{2} q_{21}-a_{1} \zeta_{1}^{2} q_{21}\right] \\
& \mu_{2}=\rho\left[\left(a_{5}-a_{3}\right) \xi_{1}^{2} q_{12}-\left(a_{1}-2 a_{4}\right) \xi_{2}^{2} q_{22}-a_{1} \zeta_{1}^{2} q_{22}\right] \\
& \boldsymbol{E}_{+}^{m}\left(X_{3}\right)=\operatorname{Diag}\left[e^{i \zeta_{1}\left(X_{3}-X_{3}^{m-1}\right)}, e^{i \zeta_{2}\left(X_{3}-X_{3}^{m-1}\right)}, e^{i \zeta_{3}\left(X_{3}-X_{3}^{m-1}\right)}\right] \\
& \boldsymbol{E}_{-}^{m}\left(X_{3}\right)=\operatorname{Diag}\left[e^{i \zeta_{1}\left(X_{3}^{m}-X_{3}\right)}, e^{i \zeta_{2}\left(X_{3}^{m}-X_{3}\right)}, e^{i \zeta_{3}\left(X_{3}^{m}-X_{3}\right)}\right]
\end{aligned}
$$

with $X_{3}^{m}$ being the $X_{3}$-coordinate of the interface between layers $m$ and $(m-1)$. Here $Q_{21}^{m}$ and $Q_{22}^{m}$ are matrices whose columns are the stress eigenvectors for the $m^{\text {th }}$ layer corresponding to wavenumbers along the 3-axis, $\zeta_{i}$ and $-\zeta_{i}$, respectively. These are obtained from the displacement eigenvectors using Eq. (2). The interface continuity conditions can then be expressed as:

where

$$
\mathbf{Q}_{-}^{m} \boldsymbol{C}^{m}=-\mathbf{Q}_{+}^{m+1} \boldsymbol{C}^{m+1}
$$

$$
\boldsymbol{Q}_{-}^{m}=\boldsymbol{Q}^{m}\left(X_{3}^{m+1}\right) \quad ; \quad \boldsymbol{Q}_{+}^{m+1}=\boldsymbol{Q}^{m+1}\left(X_{3}^{m+1}\right)
$$


These equations ensure continuity of all displacement and traction components at the interface across two layers. The surface traction conditions can be expressed as:

$$
\hat{\mathbf{Q}}_{+}^{1} \boldsymbol{C}_{+}^{1}=\boldsymbol{F} \quad ; \quad \hat{\mathbf{Q}}_{-}^{N} \boldsymbol{C}_{-}^{N}=\mathbf{0}
$$

where

$$
\hat{\mathbf{Q}}_{+}^{1}=\left[\begin{array}{ll}
-\boldsymbol{L}^{1} \mathbf{Q}_{21}^{1} & -\boldsymbol{L}^{1} \mathbf{Q}_{22}^{1} \boldsymbol{E}^{1}
\end{array}\right] ; \quad \hat{\mathbf{Q}}_{-}^{N}=\left[\begin{array}{ll}
\boldsymbol{L}^{N} \mathbf{Q}_{21}^{N} \boldsymbol{E}^{N} & \boldsymbol{L}^{N} \boldsymbol{Q}_{22}^{N}
\end{array}\right]
$$

Here the matrices $\hat{\boldsymbol{Q}}$ correspond to the lower-half of $\boldsymbol{Q}$ relating to stress. The system of equations is then solved by assembling Eqs. (19) and (20) together into a $6 N \times 6 N$ banded matrix (called the global matrix, say $\boldsymbol{G}$ ):

$$
\left[\begin{array}{ccccccccc}
\hat{\mathbf{Q}}_{+}^{1} & \mathbf{0} & \cdots & & & & & & \\
\boldsymbol{Q}_{-}^{1} & \mathbf{Q}_{+}^{2} & \mathbf{0} & \cdots & & & & & \\
& & \ddots & & & & & & \\
& \cdots & \mathbf{0} & \mathbf{Q}_{-}^{m-1} & \mathbf{Q}_{+}^{m} & \mathbf{0} & \cdots & & \\
& & \cdots & \mathbf{0} & \mathbf{Q}_{-}^{m} & \mathbf{Q}_{+}^{m+1} & \mathbf{0} & \cdots & \\
& & & & & & \ddots & & \\
& & & & & \cdots & \mathbf{0} & \mathbf{Q}_{-}^{N-1} & \mathbf{Q}_{+}^{N} \\
& & & & & & \cdots & \mathbf{0} & \hat{\mathbf{Q}}_{-}^{N}
\end{array}\right]\left[\begin{array}{c}
\boldsymbol{C}^{1} \\
\boldsymbol{C}^{2} \\
\\
\vdots \\
\boldsymbol{C}^{N}
\end{array}\right]=\left[\begin{array}{c}
\boldsymbol{F} \\
\mathbf{0} \\
\\
\vdots \\
\\
\end{array}\right]
$$

Alternatively, if the layup is symmetric about the mid-plane of the plate, then the system can be solved for the symmetric and anti-symmetric modes separately, thereby saving some computational time. The surface condition must also be split into its "symmetric" and "anti-symmetric" components. Then, the relevant surface condition on the top layer is enforced along with the continuity conditions up to the interface between layers $N / 2$ and $N / 2-1$ along with conditions of symmetry ( $u_{3}, \sigma_{32}$ and $\sigma_{31}$ being zero at the mid-plane) or anti-symmetry $\left(u_{1}, u_{2}\right.$ and $\sigma_{33}$ being zero at the mid-plane). The problem is thus reduced to two systems, each of complexity $3 N \times 3 N$. With the problem constraints now enforced, if the forcing function is also known, this equation can be solved to find the constants, $C^{m}$.

\section{Forcing function due to piezo-actuator}

The piezo actuator is modeled as causing in-plane shear traction of uniform magnitude (say $\tau_{0}$ per unit length) along its perimeter, in the direction normal to the free edge on the plate surface $X_{3}=0$ (see Figure 2). In this model, the dynamics of the actuator are neglected and it is assumed that the plate dynamics are uncoupled from the actuator dynamics. This model was proposed by Crawley and de Luis ${ }^{17}$ to describe quasi-static induced strain actuation of beams by surface-bonded piezo-actuators. For that case, they proved that the model is a good approximation if the actuator thickness is small compared to that of the substrate and the bond layer is thin and stiff. This was also proven to be a good assumption in the authors' earlier work for isotropic plates². In this work, two specific shapes of the piezo-actuator are considered: rectangular and ring-shaped. These are the most commonly used shapes in GW SHM.

For the ring-shaped actuator located at the center of the coordinate system, the actuation components $f_{i}, i=1$ to 3 and their respective 2-D spatial Fourier transforms $F_{i}$ are given by:

$$
\begin{array}{ll}
f_{1}=\left(\delta\left(R-A_{o}\right)-\delta\left(R-A_{i}\right)\right) \cos \Theta & F_{1}=-i\left(A_{o} J_{1}\left(K A_{o}\right)-A_{i} J_{1}\left(K A_{i}\right)\right) K_{1} / K \\
f_{2}=\left(\delta\left(R-A_{o}\right)-\delta\left(R-A_{i}\right)\right) \sin \Theta & F_{2}=-i\left(A_{o} J_{1}\left(K A_{o}\right)-A_{i} J_{1}\left(K A_{i}\right)\right) K_{2} / K \\
f_{3}=0 & F_{3}=0
\end{array}
$$

where $A_{i}$ is the inner radius of the ring-shaped actuator and $A_{o}$ is the outer radius. $J_{1}()$ is the Bessel function of the first kind and order one. $\delta()$ is the Dirac-delta function. $R$ and $\Theta$ are the polar spatial coordinates, i.e., $R=\sqrt{\left(X_{1}^{2}+X_{2}^{2}\right)}$ and $\Theta=\tan ^{-1}\left(X_{2} / X_{1}\right)$ and $K$ and $\Gamma$ are polar wavenumber coordinates, i.e., $K=\sqrt{\left(K_{1}^{2}+K_{2}^{2}\right)}$ and $\Gamma=\tan ^{-1}\left(K_{2} / K_{1}\right)$. Similarly, for the rectangular actuator of dimensions $2 A_{1} \times 2 A_{2}$ (along the $X_{1}$ - and $X_{2}$-axes respectively), which is located at the center of the coordinate system:

$$
\begin{aligned}
& f_{1}=\tau_{0}\left(\delta\left(X_{1}-A_{1}\right)-\delta\left(X_{1}+A_{1}\right)\right)\left(H e\left(X_{2}+A_{2}\right)-H e\left(X_{2}-A_{2}\right)\right) \\
& F_{1}=-4 \tau_{0} \sin \left(K_{1} A_{1}\right) \sin \left(K_{2} A_{2}\right) / i K_{2} \\
& f_{2}=\tau_{0}\left(H e\left(X_{1}+A_{1}\right)-H e\left(X_{1}-A_{1}\right)\right)\left(\delta\left(X_{2}-A_{2}\right)-\delta\left(X_{2}+A_{2}\right)\right) \\
& F_{2}=-4 \tau_{0} \sin \left(K_{1} A_{1}\right) \sin \left(K_{2} A_{2}\right) / i K_{1} \\
& f_{3}=0 \quad ; \quad F_{3}=0
\end{aligned}
$$




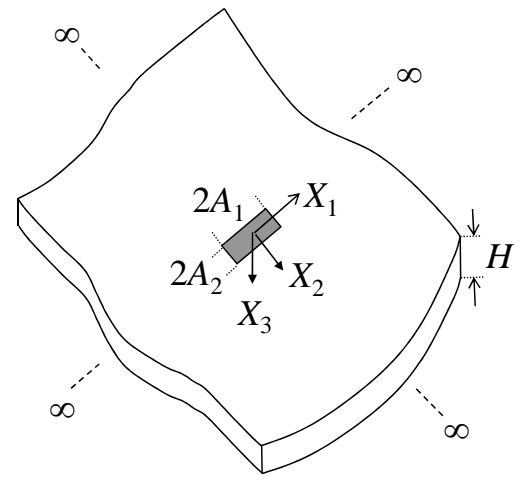

(a)



(b)

Figure 4. Configurations considered: (a) rectangular and (b) ring-shaped actuators

where $\mathrm{He}$ ( ) is the Heaviside function. The constants in Eq. (21) can then be analytically solved using Cramer's rule:

where

$$
\begin{aligned}
C_{1+}^{1} & =\frac{N^{1}(K, \Gamma)}{\Delta(K, \Gamma)}, \text { etc., } \\
N^{1}(K, \Gamma) & =\operatorname{det}\left[\begin{array}{cccccccccc}
\boldsymbol{F} & \mathbf{0} & \cdots & & & & & & \\
\mathbf{0} & \boldsymbol{Q}_{+}^{2} & \mathbf{0} & \cdots & & & & & \\
& & \ddots & & & & & & \\
& \cdots & \mathbf{0} & \boldsymbol{Q}_{-}^{m-1} & \boldsymbol{Q}_{+}^{m} & \mathbf{0} & \cdots & & \\
\vdots & & \cdots & \mathbf{0} & \boldsymbol{Q}_{-}^{m} & \boldsymbol{Q}_{+}^{m+1} & \mathbf{0} & \cdots & \\
\Delta(K, \Gamma) & =\operatorname{det}(\boldsymbol{G}) & & & & & & \ddots & & \\
\mathbf{0} & & & & & & \cdots & \mathbf{0} & \hat{\boldsymbol{Q}}_{-}^{N}
\end{array}\right]
\end{aligned}
$$

D. Spatial Fourier integral inversion

With the constants known, the expression for displacement in the wavenumber domain can be obtained from Eqs. (17) and (24). The Fourier inversion formula, Eq. (15), can then be used to recover the spatial domain solution. For the case of a rectangular actuator, this leads to an expression of the following form for displacement along the 1direction in the spatial domain:

$$
u_{1}\left(X_{1}, X_{2}, 0\right)=\int_{0}^{2 \pi} \int_{0}^{\infty} \frac{-\tau_{0}}{i \pi^{2}} \sin \left(K A_{1} \cos \Gamma\right) \sin \left(K A_{2} \sin \Gamma\right) \frac{N(K, \Gamma)}{\Delta(K, \Gamma)} e^{-i\left(K\left(X_{1} \cos \Gamma+X_{2} \sin \Gamma\right)-\omega t\right)} K d K d \Gamma
$$

To solve the inverse Fourier integral, the residue theorem from complex analysis is used for the integral along the $K$ direction. For convenience, the integral in Eq. (25) is rewritten as:

$$
\begin{aligned}
u_{1}\left(X_{1}, X_{2}, 0\right)=\int_{0}^{2 \pi} \int_{0}^{\infty}\left(e^{i K A_{1} \cos \Gamma}-e^{-i K A_{1} \cos \Gamma}\right) & \cdot\left(e^{i K A_{2} \sin \Gamma}-e^{-i K A_{2} \sin \Gamma}\right) \\
& \times \frac{\tau_{0}}{4 i \pi^{2}} \cdot \frac{N(K, \Gamma)}{\Delta(K, \Gamma)} e^{-i\left(K\left(X_{1} \cos \Gamma+X_{2} \sin \Gamma\right)-\omega t\right)} K d K d \Gamma
\end{aligned}
$$




$$
\begin{aligned}
u_{1}\left(X_{1}, X_{2}, 0\right)= & \int_{0}^{2 \pi} \int_{0}^{\infty} \frac{\tau_{0}}{4 i \pi^{2}} \cdot \frac{N(K, \Gamma)}{\Delta(K, \Gamma)} e^{-i\left(K\left(\left(X_{1}-A_{1}\right) \cos \Gamma+\left(X_{2}-A_{2}\right) \sin \Gamma\right)-\omega t\right)} K d K d \Gamma+ \\
& +\int_{0}^{2 \pi} \int_{0}^{\infty} \frac{-\tau_{0}}{4 i \pi^{2}} \cdot \frac{N(K, \Gamma)}{\Delta(K, \Gamma)} e^{-i\left(K\left(\left(X_{1}-A_{1}\right) \cos \Gamma+\left(X_{2}+A_{2}\right) \sin \Gamma\right)-\omega t\right)} K d K d \Gamma+ \\
& +\int_{0}^{2 \pi} \int_{0}^{\infty} \frac{-\tau_{0}}{4 i \pi^{2}} \cdot \frac{N(K, \Gamma)}{\Delta(K, \Gamma)} e^{-i\left(K\left(\left(X_{1}+A_{1}\right) \cos \Gamma+\left(X_{2}-A_{2}\right) \sin \Gamma\right)-\omega t\right)} K d K d \Gamma+ \\
& +\int_{0}^{2 \pi} \int_{0}^{\infty} \frac{\tau_{0}}{4 i \pi^{2}} \cdot \frac{N(K, \Gamma)}{\Delta(K, \Gamma)} e^{-i\left(K\left(\left(X_{1}+A_{1}\right) \cos \Gamma+\left(X_{2}+A_{2}\right) \sin \Gamma\right)-\omega t\right)} K d K d \Gamma
\end{aligned}
$$

First, consider the first of the four integrals in the second line of Eq. (26), say $I_{1}$, which corresponds to $\left(a_{1}\right.$, $\left.a_{2}\right)$. It is further rewritten as follows:

where

$$
\begin{aligned}
& I_{1}=\int_{\tilde{\Theta}_{1}-\pi / 2}^{\tilde{\Theta}_{1}+\pi / 2} \int_{-\infty}^{\infty} \frac{\tau_{0}}{4 i \pi^{2}} \cdot \frac{N(K, \Gamma)}{\Delta(K, \Gamma)} e^{-i\left(K \tilde{R}_{1} \cos \left(\Gamma-\tilde{\Theta}_{1}\right)-\omega t\right)} K d K d \Gamma \\
& \tilde{\Theta}_{1}=\tan ^{-1}\left(\frac{X_{2}-A_{2}}{X_{1}-A_{1}}\right) ; \quad \tilde{R}_{1}=\sqrt{\left(X_{1}-A_{1}\right)^{2}+\left(X_{2}-A_{2}\right)^{2}}
\end{aligned}
$$

This ensures that the coefficient of $K$ in the complex exponential remains positive over the domain of integration. The inner integral along the real $K$-axis is replaced by a contour integral in the complex $K$-plane, the semi-circular portion of which has radius $|K| \rightarrow \infty$ (see Figure 5). The integrand is singular at the roots $\hat{K}$ of $\Delta(K, \Gamma)=0$, which is the dispersion equation for the multilayered composite plate. These roots are the allowable in-plane radial wavenumbers for the multilayered composite plate at angular frequency $\omega . \Delta(K, \Gamma)$ is symmetric about the $K$-axis. Therefore, if $\hat{K}$ is a root of $\Delta(K, \Gamma)=0$, then so is $-\hat{K}$. However, since only the outgoing wave is desired, the negative roots are not included in the contour. Using the residue theorem for the inner integral in Eq. (26) yields in this case (assuming $I$ is the integrand in $I_{1}$ ):

$$
\int_{-\infty}^{\infty} I d K+\int_{C} I d K=-\pi i \sum_{\hat{K}} \operatorname{Res}(\mathrm{I}(\hat{K}))
$$

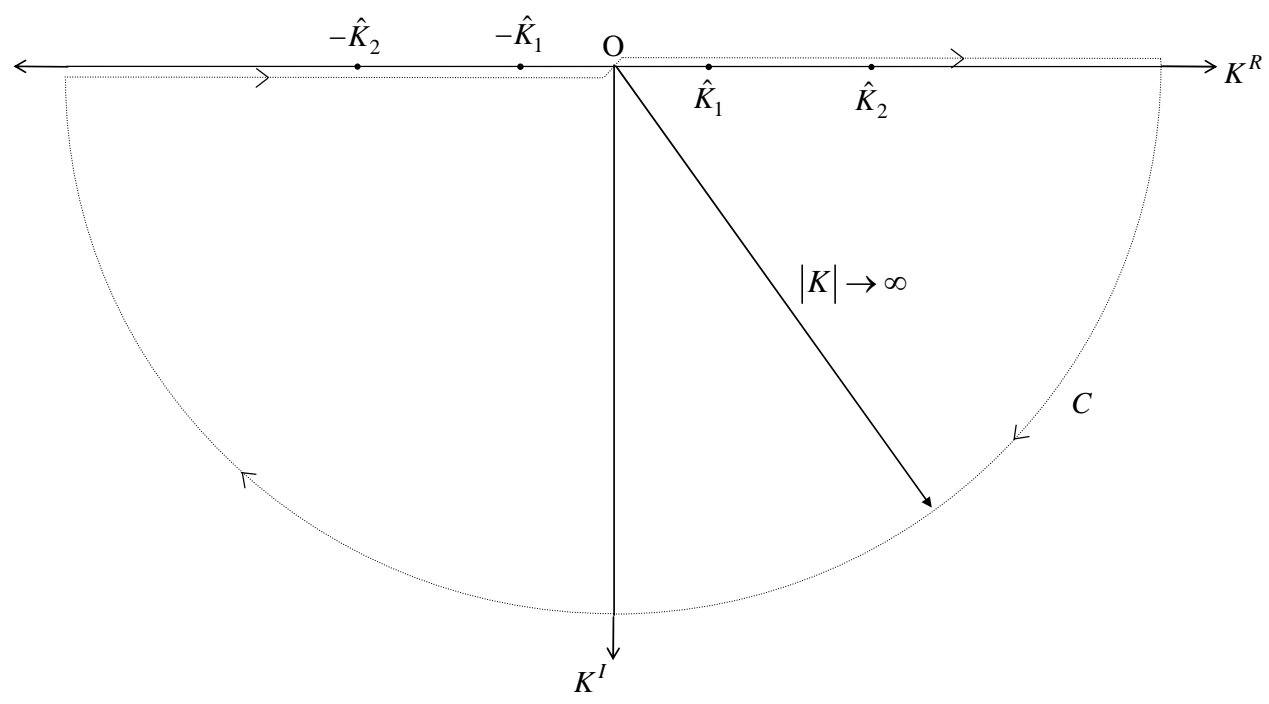

Figure 5. Contour integral in the complex $K$-plane to invert the displacement integrals using residue theory 
It remains to be shown that the contribution from $C$ vanishes. As explained in Miklowitz ${ }^{18}$ for a similar plane wave excitation problem, $(K \cdot N(K, \Gamma)) / \Delta(K, \Gamma)$ for large $|K|$ is of order $1 / K$, and therefore tends to zero as $|K| \rightarrow \infty$. Furthermore, along $C$, if $K=K^{R}-i K^{I} ; \quad K^{R}, K^{I}>0$ :

$$
\left|e^{-i\left(K \tilde{R}_{1} \cos \left(\Gamma-\tilde{\Theta}_{1}\right)-\omega t\right)}\right|=\left|e^{i \omega t} \cdot e^{-i K^{R} \tilde{R}_{1} \cos \left(\Gamma-\tilde{\Theta}_{1}\right)} \cdot e^{-K^{l} \tilde{R}_{1} \cos \left(\Gamma-\tilde{\Theta}_{1}\right)}\right| \leq\left|e^{-K^{I} \tilde{R}_{1} \cos \left(\Gamma-\tilde{\Theta}_{1}\right)}\right|
$$

Since $\tilde{R}_{1}$ and $\cos \left(\Gamma-\tilde{\Theta}_{1}\right)$ are both always positive, the term $\left|e^{-K^{I} \tilde{R}_{1} \cos \left(\Gamma-\Theta_{1}\right)}\right|$ is finite and is bounded by zero as $K^{I} \rightarrow \infty$. Therefore:

$$
\begin{aligned}
& \int_{C} I d K=0 ; \quad I_{1}=\int_{-\infty}^{\infty} I d K=-\pi i \sum_{\hat{K}} \operatorname{Res}(\mathrm{I}(\hat{K})) \\
& I_{1}=\sum_{\hat{K}} \int_{\tilde{\Theta}_{1}-\pi / 2}^{\tilde{\Theta}_{1}+\pi / 2} \frac{-\tau_{0}}{4 \pi} \cdot \frac{N(\hat{K}, \Gamma)}{\Delta^{\prime}(\hat{K}, \Gamma)} e^{-i\left(\hat{K} \tilde{R}_{1} \cos \left(\Gamma-\tilde{\Theta}_{1}\right)-\omega t\right)} d \Gamma
\end{aligned}
$$

where ()$^{\prime}$ indicates derivative with respect to $K$. Similar analysis can be used to solve the other three integrals in Eq. (26), to finally yield the expression for $u_{1}$ :

$$
\begin{aligned}
u_{1}= & \sum_{\hat{K}} \int_{\tilde{\Theta}_{1}-\pi / 2}^{\tilde{\Theta}_{1}+\pi / 2} \frac{-\tau_{0}}{4 \pi} \cdot \frac{N(\hat{K}, \Gamma)}{\Delta^{\prime}(\hat{K}, \Gamma)} e^{-i\left(\hat{K} \tilde{R}_{1} \cos \left(\Gamma-\tilde{\Theta}_{1}\right)-\omega t\right)} d \Gamma+\sum_{\hat{K}} \int_{\tilde{\Theta}_{2}-\pi / 2}^{\tilde{\Theta}_{2}+\pi / 2} \frac{\tau_{0}}{4 \pi} \cdot \frac{N(\hat{K}, \Gamma)}{\Delta^{\prime}(\hat{K}, \Gamma)} e^{-i\left(\hat{K} \tilde{R}_{2} \cos \left(\Gamma-\tilde{\Theta}_{2}\right)-\omega t\right)} d \Gamma+ \\
& +\sum_{\hat{K}} \int_{\tilde{\Theta}_{3}-\pi / 2}^{\tilde{\Theta}_{3}+\pi / 2} \frac{\tau_{0}}{4 \pi} \cdot \frac{N(\hat{K}, \Gamma)}{\Delta^{\prime}(\hat{K}, \Gamma)} e^{-i\left(\hat{K} \tilde{R}_{3} \cos \left(\Gamma-\tilde{\Theta}_{3}\right)-\omega t\right)} d \Gamma+\sum_{\hat{K}} \int_{\tilde{\Theta}_{4}-\pi / 2}^{\tilde{\Theta}_{4}+\pi / 2} \frac{-\tau_{0}}{4 \pi} \cdot \frac{N(\hat{K}, \Gamma)}{\Delta^{\prime}(\hat{K}, \Gamma)} e^{-i\left(\hat{K} \tilde{R}_{4} \cos \left(\Gamma-\tilde{\Theta}_{4}\right)-\omega t\right)} d \Gamma
\end{aligned}
$$

This procedure can also be used for the other displacement components. An approximate closed form solution can be obtained for the far field using the method of stationary phase. This is assuming damping is not modeled and that the integrand is real-valued. If damping is modeled, then a similar approximation can be done using the method of steepest descent ${ }^{19}$. As explained in Graff ${ }^{19}$, for large $r$ :

$$
\int_{\psi_{1}}^{\psi_{2}} f(\psi) e^{i r h(\psi)} d \psi \approx \sqrt{\frac{2 \pi}{r h^{\prime \prime}\left(\psi_{0}\right)}} f\left(\psi_{0}\right) e^{i\left(r \psi_{0}+\pi / 4\right)}
$$

Therefore, for large values of $R$ (which leads to large values of $\tilde{R}_{k}, k=1$ to 4 ):

$$
\begin{aligned}
& u_{1}=\sum_{\hat{K}} \sqrt{\frac{2 \pi}{\left.\tilde{R}_{1} \cdot \frac{d^{2}\left(\hat{K} \cos \left(\Gamma-\tilde{\Theta}_{1}\right)\right)}{d \Gamma^{2}}\right|_{\Gamma=\bar{\Gamma}_{1}}}} \frac{-\tau_{0} N\left(\hat{K}, \bar{\Gamma}_{1}\right)}{4 \pi \Delta^{\prime}\left(\hat{K}, \bar{\Gamma}_{1}\right)} e^{-i\left(\hat{K} \tilde{R}_{1} \cos \left(\bar{\Gamma}_{1}-\tilde{\Theta}_{1}\right)-\omega t+\pi / 4\right)}+ \\
& +\sum_{\hat{K}} \sqrt{\frac{2 \pi}{\left.\tilde{R}_{2} \cdot \frac{d^{2}\left(\hat{K} \cos \left(\Gamma-\tilde{\Theta}_{2}\right)\right)}{d \Gamma^{2}}\right|_{\Gamma=\bar{\Gamma}_{2}}}} \frac{\tau_{0} N\left(\hat{K}, \bar{\Gamma}_{2}\right)}{4 \pi \Delta^{\prime}\left(\hat{K}, \bar{\Gamma}_{2}\right)} e^{-i\left(\hat{K} \tilde{K}_{2} \cos \left(\bar{\Gamma}_{2}-\tilde{\Theta}_{2}\right)-\omega t+\pi / 4\right)}+
\end{aligned}
$$

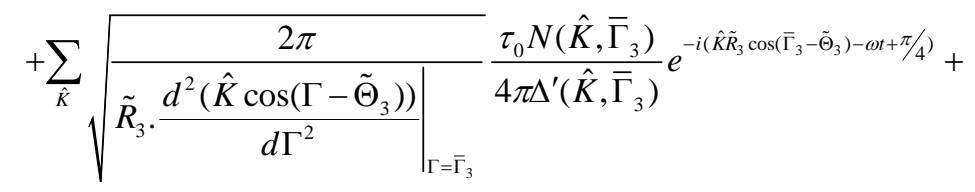



where $\tan \left(\bar{\Gamma}_{k}-\tilde{\Theta}_{k}\right)=\frac{1}{\hat{K}} \frac{d \hat{K}}{d \Gamma}$. Thus, $\left(\bar{\Gamma}_{k}-\tilde{\Theta}_{k}\right)$ is the angle between the phase velocity and group velocity vectors ${ }^{16}$. This implies that the contributions from $\bar{\Gamma}_{k}$ dominate the integrals over $\Gamma$ at large distances from the source. This 
reiterates a well-known fact about wave propagation in composites, i.e., the wave travels at a "steering angle," which may be different from the angle that it was launched along by its source.

An analogous solution can be obtained for the ring-shaped actuators. In this case, an expression of the following form for displacement along the 1-direction in the spatial domain is obtained:

$$
u_{1}\left(X_{1}, X_{2}, 0\right)=\int_{0}^{2 \pi} \int_{0}^{\infty}\left(A_{o} J_{1}\left(K A_{o}\right)-A_{i} J_{1}\left(K A_{i}\right)\right) \frac{\tau_{0}}{4 \pi^{2}} \cdot \frac{N(K, \Gamma)}{\Delta(K, \Gamma)} e^{-i\left(K\left(X_{1} \cos \Gamma+X_{2} \sin \Gamma\right)-\omega t\right)} K d K d \Gamma
$$

For simplicity of analysis, consider the case of a circular actuator $\left(A_{i}=0\right)$. The integral in Eq. (35) is rewritten thus:

$$
\begin{aligned}
u_{1}(R, \Theta, 0) & =\int_{\Theta-\pi / 2}^{\Theta+\pi / 2} \int_{-\infty}^{\infty} A_{o} J_{1}\left(K A_{o}\right) \frac{\tau_{0}}{4 \pi^{2}} \cdot \frac{N(K, \Gamma)}{\Delta(K, \Gamma)} e^{-i(K R \cos \Theta-\omega t)} K d K d \Gamma \\
& =\int_{\Theta-\pi / 2}^{\Theta+\pi / 2} \int_{-\infty}^{\infty} A_{o}\left(H_{1}^{(1)}\left(K A_{o}\right)+H_{1}^{(2)}\left(K A_{o}\right)\right) \frac{\tau_{0}}{8 \pi^{2}} \cdot \frac{N(K, \Gamma)}{\Delta(K, \Gamma)} e^{-i(K R \cos \Theta-\omega t)} K d K d \Gamma
\end{aligned}
$$

This is then re-arranged as follows:

$$
\begin{aligned}
u_{1}(R, \Theta, 0)= & \int_{\Theta-\pi / 2}^{\Theta+\pi / 2} \int_{-\infty}^{\infty} A_{o} H_{1}^{(2)}\left(K A_{o}\right) \frac{\tau_{0}}{8 \pi^{2}} \cdot \frac{N(K, \Gamma)}{\Delta(K, \Gamma)} e^{-i(K R \cos \Theta-\omega t)} K d K d \Gamma+ \\
& +\int_{\Theta-\pi / 2}^{\Theta+\pi / 2} \int_{-\infty}^{\infty} A_{o} H_{1}^{(1)}\left(K A_{o}\right) \frac{\tau_{0}}{8 \pi^{2}} \cdot \frac{N(K, \Gamma)}{\Delta(K, \Gamma)} e^{-i(K R \cos \Theta-\omega t)} K d K d \Gamma
\end{aligned}
$$

As before, for each of the two integrals, residue calculus is used. The contours must be chosen such that the integrands remain finite-valued. Over the semi-circular contour $C$ :

$$
|K| \rightarrow \infty \therefore H_{1}^{(2)}\left(K A_{0}\right) \approx \sqrt{2 / \pi K A_{0}} e^{-i\left(K A_{0}-3 \pi / 2\right)} ; H_{1}^{(1)}\left(K A_{0}\right) \approx \sqrt{2 / \pi K A_{0}} e^{i\left(K A_{0}-3 \pi / 2\right)}
$$

Therefore, for the first integral, over $C$, the integrand in the first integral of Eq. (37) is approximately:

$$
\sqrt{2 / \pi K A_{o}} e^{-i\left(K\left(R \cos \Theta+A_{0}\right)-3 \pi / 2\right)} \frac{N(K, \Gamma)}{\Delta(K, \Gamma)} K
$$

Therefore, the coefficient of $K$ in the exponent always remains positive and the contour can be closed in the lower half-plane over the full angular range, as was done for the rectangular actuator. However, for the second integral of Eq. (37), over $C$, the integrand is approximately:

$$
\sqrt{2 / \pi K A_{0}} e^{-i\left(K\left(R \cos \Theta-A_{0}\right)-3 \pi / 2\right)} \frac{N(K, \Gamma)}{\Delta(K, \Gamma)} K
$$

In this case, the coefficient of $K$ in the exponent is negative over part of angular range (defined by $\cos \Theta<A_{o} / R$ ). Therefore, over this part of the angular range, the contour must be closed in the upper half of the complex $K$-plane. This results in a positive sign for the residue over that part of the angular range unlike earlier when closing in the lower half plane resulted in a negative sign for the residue.

\section{Implementation of the Formulation and Results for Harmonic Excitation}

The theoretical formulation described above was implemented in Fortran 90. The linear algebra package LAPACK $^{20}$ for Fortran 90 was employed to evaluate the determinants of large banded matrices. The roots of the dispersion equation $\Delta(K, \Gamma, 0)=0$ were simply computed by the "zero-crossing" approach, i.e., by evaluating the determinant of the matrix over a fine grid in the $(K, \Gamma)$ plane and looking for sign changes in the value of the determinant. In doing so, one has to avoid the bulk wave velocities of the composite material, which are also roots of the dispersion equation. One also has to take care to use double-precision variables and compute the roots with high precision since with the very large matrices involved, small errors in the values of the roots cause large errors in the response solution. The code implemented in Fortran 90 was computationally efficient, with each run being completed in a few minutes on a standard desktop computer (1.2 GHz Pentium IV with 256 MB RAM).

Some results from the proposed formulation are presented here. First, a unidirectional graphite-epoxy composite plate (material properties: $\rho=1578 \mathrm{~kg} / \mathrm{m}^{3} ; c_{11}=160.73 \mathrm{GPa}, c_{12}=6.44 \mathrm{GPa}, c_{22}=13.92 \mathrm{GPa}, c_{23}=6.92 \mathrm{GPa}, c_{55}$ $=7.07 \mathrm{GPa}$, this is the same material used in Ref. 9) was analyzed. Damping was not considered for these analyses. The slowness surfaces (plots of inverse of phase velocity versus propagation direction) for the $A_{0}$ and $S_{0}$ modes at 
$500 \mathrm{kHz}$ are shown in Figure 1 (b). The out-of-plane harmonic surface displacement plots over a quarter section of this unidirectional plate at $500 \mathrm{kHz}$ due to a 1 -cm diameter circular actuator surface-bonded at the center $\left(\mathrm{S}_{0}\right.$ mode) is shown in Figure 6. There is uniform actuation along all directions and the actuator diameter is equal to the halfwavelength along the $0^{\circ} / 180^{\circ}$ direction, which is close to the optimal size along that direction (actuator size optimization is discussed in more detail for isotropic structures in the authors' earlier work for isotropic plates ${ }^{2}$ ). Despite this, and the fact that the predominant direction of the group velocity vector is along the $0^{\circ} / 180^{\circ}$ direction, there is a strong preference for radiation along the $90^{\circ} / 270^{\circ}$ direction, normal to the fiber direction. (The group velocity vector is normal to the slowness surface.) This is logical, since the composite plate is much less stiff along this direction, thereby offering less impedance to the GW field at $90^{\circ} / 270^{\circ}$ compared to $0^{\circ} / 180^{\circ}$. This is evident in the semi-log plot of the kernel function $N / \Delta^{\prime}$ versus the propagation angle (Figure 7), which relates to the excitability of the GW field along different propagation directions in the composite plate. There is a difference of three orders of magnitude between the excitability along $90^{\circ}$ and $0^{\circ}$ (the local valleys between $90^{\circ}$ and $0^{\circ}$ are possibly due to structural anti-resonances). The directionality can only be weakly controlled by actuator design, as illustrated in Figure 8. In this configuration, the actuator dimension along the $0^{\circ} / 180^{\circ}$ direction is still equal to the halfwavelength along this direction. However, the actuator dimension along the $90^{\circ} / 270^{\circ}$ direction is equal to two times the wavelength along this direction, which nullifies the radiation emerging from the actuator along it. However, the GWs launched at other angles tend to "steer" towards a direction close to $90^{\circ} / 270^{\circ}$. Similar harmonic plots for the $\mathrm{A}_{0}$ mode at $500 \mathrm{kHz}$ are shown in Figure 9 for a circular actuator and in Figure 10 for a rectangular actuator. The dimension of the circular actuator is "optimal" approximately at $48^{\circ}$ relative to the fiber direction, which results in the peak amplitude occurring roughly at that polar angle along the actuator perimeter. However, again due to steering, the GW field is predominantly along $90^{\circ} / 270^{\circ}$. The dimensions of the rectangular actuator in Figure 10 were again chosen to maximize radiation along $0^{\circ} / 180^{\circ}$ and minimize radiation along $90^{\circ} / 270^{\circ}$.

Analysis was also done for plates with different quasi-isotropic layups of the same graphite-epoxy material. The first layup was [0/45/-45/90]s, with each ply being 0.11-mm thick. For this configuration, up to around $500 \mathrm{kHz}$, the $\mathrm{S}_{0}$ mode is practically isotropic. The slight variations in wavespeed with direction are imperceptible to the naked eye (e.g., see Figure 11). In addition, when excited by a circular actuator, the harmonic GW field seems to be uniform across different directions (shown in Figure $13 \mathrm{for} 200 \mathrm{kHz}$ ) for the $S_{0}$ mode in this quasi-isotropic layup. For the $\mathrm{A}_{0}$ mode at $200 \mathrm{kHz}$, the harmonic GW field seems to be focused along two directions in the far-field (see Figure 14), which are the predominant directions of the group velocity vector over the $360^{\circ}$ angular range. The layup [0/90/45/-45] s was also analyzed for the $A_{0}$ mode. The slowness surfaces of both layups are shown in Figure 12 for the $\mathrm{A}_{0}$ mode (at $200 \mathrm{kHz}$ ). In this case, the slowness curve is closer to being isotropic and, therefore, the GW field tends to be more uniform over the angular span in the far-field (see Figure 15). There is some mild tendency of the group velocity vectors to be along the $0^{\circ} / 180^{\circ}$ and $90^{\circ} / 270^{\circ}$ directions, which possibly explains the slightly stronger GW amplitude along these directions in the far-field.
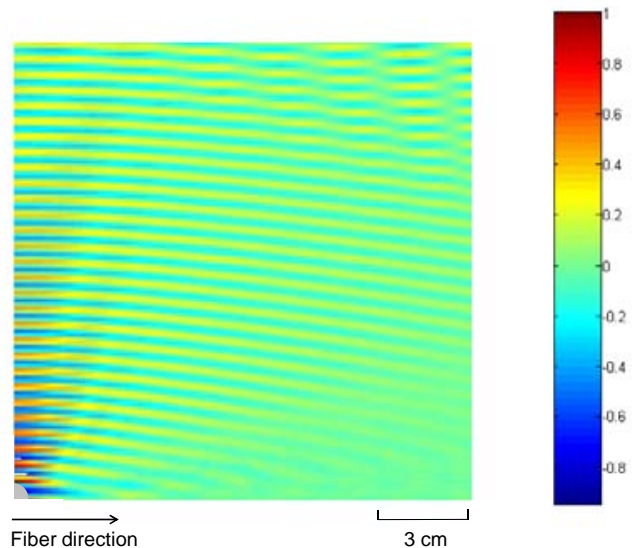

Figure 6. Harmonic radiation plot $\left(u_{3}\right)$ over a quarter-section of a 1-mm unidirectional graphiteepoxy plate $\left(S_{0}\right.$ mode) at $500 \mathrm{kHz}$ due to excitation by a 1-cm diameter actuator (lower-left corner, gray)

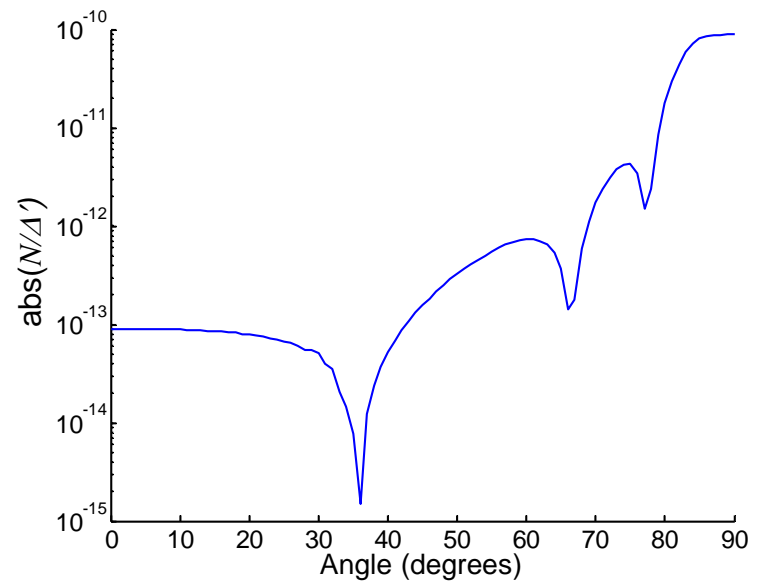

Figure 7. Variation of the kernel function $N / \Delta^{\prime}$ with propagation angle at $500 \mathrm{kHz}, S_{0}$ mode in the $1-\mathrm{mm}$ thick unidirectional graphite epoxy plate 




Figure 8. Harmonic radiation plot $\left(u_{3}\right)$ over a quarter-section of a 1-mm unidirectional graphiteepoxy plate ( $S_{0}$ mode) at $500 \mathrm{kHz}$ due to excitation by a rectangular actuator of dimensions $a_{1}=0.5 \mathrm{~cm}, a_{2}=$ $2 \mathrm{~cm}$ (in gray, lower-left corner).

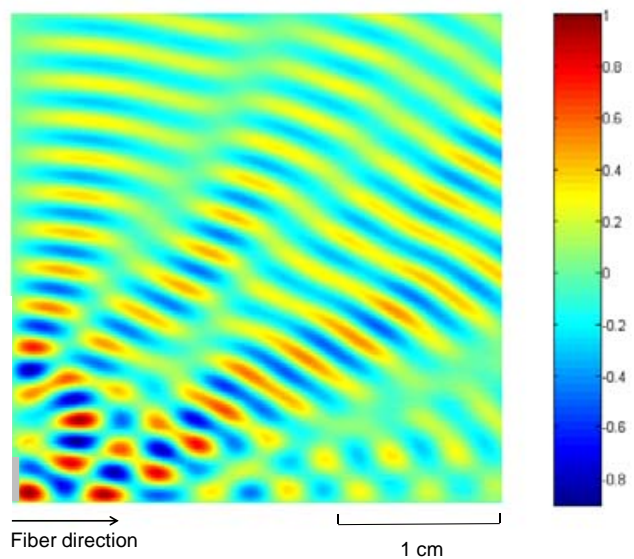

Figure 10. Harmonic radiation plot $\left(u_{3}\right)$ over a quarter-section of a 1-mm unidirectional graphiteepoxy plate ( $A_{0}$ mode) at $500 \mathrm{kHz}$ due to excitation by a rectangular actuator of dimensions $a_{1}=0.055 \mathrm{~cm}, a_{2}$ $=0.36 \mathrm{~cm}$ (in gray, lower-left corner).

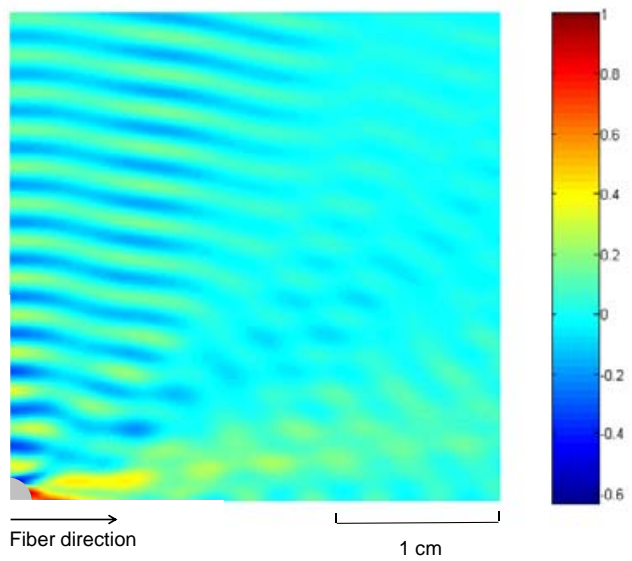

Figure 9. Harmonic radiation plot $\left(u_{3}\right)$ over a quarter-section of a 1-mm unidirectional graphiteepoxy plate ( $A_{0}$ mode) at $500 \mathrm{kHz}$ due to excitation by a 0.3-cm diameter actuator (at the lower-left corner, in gray)

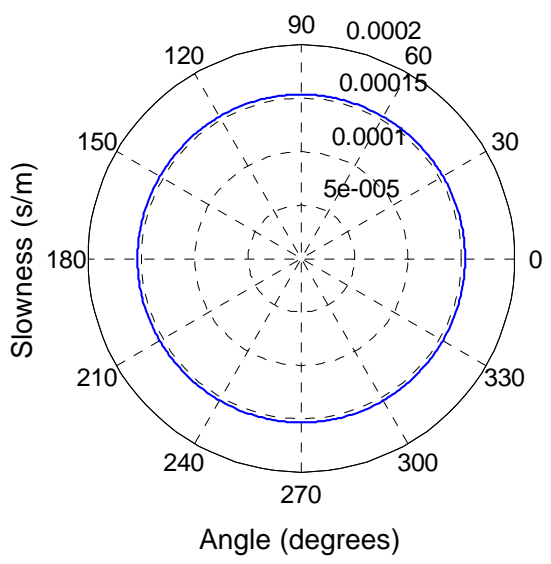

Figure 11. Slowness surface for $S_{0}$ mode in a quasiisotropic ([0/45/-45/90]s, with each ply being $0.11-\mathrm{mm}$ thick) graphite-epoxy composite plate at $200 \mathrm{kHz}$.

\section{Concluding Remarks}

This work addressed the modeling of the guided-wave (GW) field excited by finite-dimensional surface-bonded piezoelectric wafer transducers in multi-layered composite plates. The objective was to use the models to support the design of GW structural health monitoring (SHM) systems using this class of transducers for composite structures. The individual layers were assumed to be transversely isotropic, and the actuators were treated as causing shear traction along their free edges on the plate surface (assuming uncoupled actuator-substrate dynamics). The threedimensional governing equations for the composite substrate were used, and the global matrix approach was adopted to enforce surface and interfacial conditions, thereby capturing all possible GW modes without using reduced structural formulations. Expressions for the specific shapes of rectangular and ring-shaped piezo-actuators were derived. A rigorous Fourier inversion procedure was outlined to extract the outgoing wave solution. The numerical implementation of the developed formulation was described. Sample results from analysis done on some configurations were then presented. For unidirectional composites, it was observed that the direction normal to the fiber direction $\left(90^{\circ}\right)$ was the preferential direction of GW radiation, due to the lowest impedance along it. By appropriately designing rectangular actuators, this tendency can be somewhat controlled close to the source. 
However, in the far-field, the GW tends to steer back to a direction close to the least impedance one. For quasiisotropic layups, the $\mathrm{S}_{0}$ mode is approximately isotropic at frequency-plate thickness products up to around 500 $\mathrm{kHz}-\mathrm{mm}$ while the $\mathrm{A}_{0}$ mode tends to steer towards the directions along which the group velocity vector is dominant. Future experiments are planned with graphite-epoxy plates to further investigate the phenomenon.



Figure 12. Slowness surfaces for $A_{0}$ mode in graphite-epoxy plates with quasi-isotropic layups (with each ply being $0.11-\mathrm{mm}$ thick), at $200 \mathrm{kHz}$.


Figure 14. Harmonic radiation plot $\left(u_{3}\right)$ over a halfsection of the quasi-isotropic $\left([0 / 45 /-45 / 90]_{s}\right)$ graphiteepoxy plate at $200 \mathrm{kHz}\left(\mathrm{A}_{0}\right.$ mode) due to excitation by a 0.25-cm dia. circular actuator (in gray).
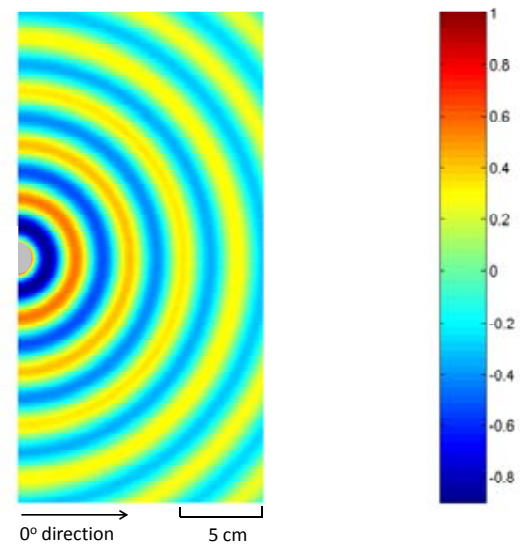

Figure 13. Harmonic radiation plot $\left(u_{3}\right)$ over a halfsection of the quasi-isotropic $\left([0 / 45 /-45 / 90]_{s}\right)$ graphiteepoxy plate at $200 \mathrm{kHz}\left(\mathrm{S}_{0}\right.$ mode) due to excitation by a 1.6-cm dia. circular actuator (in gray).


Figure 15. Harmonic radiation plot $\left(u_{3}\right)$ over a halfsection of the quasi-isotropic $\left([0 / 90 / 45 /-45]_{s}\right)$ graphiteepoxy plate at $200 \mathrm{kHz}\left(\mathrm{A}_{0}\right.$ mode) due to excitation by a $0.25-\mathrm{cm}$ dia. circular actuator (in gray).

\section{Acknowledgment}

This work is supported by the Space Vehicle Technology Institute under grant NCC3-989 jointly funded by NASA and DoD within the NASA Constellation University Institutes Project, with Ms. Claudia Meyer as the project manager.

\section{References}

1 Raghavan A. and Cesnik C.E.S., "Review of guided-wave structural health monitoring," The Shock and Vibration Digest, Vol. 39, 2007, pp. 91-114

2 Raghavan A. and Cesnik C.E.S., "Finite dimensional piezoelectric transducer modeling for guided wave based structural health monitoring," Smart Materials and Structures, Vol. 14, 2005, pp. 1448-1461

3 Raghavan A. and Cesnik C.E.S., "3-D elasticity-based modeling of anisotropic piezocomposite transducers for guided wave structural health monitoring," to appear in ASME Journal of Vibration and Acoustics (special issue on damage 
detection and structural health monitoring), 2007

4 Rose J.L., Ultrasonic waves in solid media, Cambridge University Press, Cambridge, UK, 1999

5 Lamb H., “On waves in an elastic plate,” Proc. Royal Society of London Series A, Vol. 93, No. 651, 1917, pp. 293-312

6 Gazis D.C., "Exact analysis of the plane-strain vibrations of thick-walled hollow cylinders,” Journal of the Acoustical Society of America, Vol. 30, 1958, pp. 786-794

7 Ditri J., Rose J.L., "Excitation of guided waves in generally anisotropic layers using finite sources,” Journal of Applied Mechanics (Transactions of the ASME), Vol. 61, No. 2, 1994, pp. 330-338

8 Mal A.K., "Wave propagation in layered composite laminates under periodic surface loads,” Wave Motion, Vol. 10, 1988, pp. 257-266

9 Lih S.-S. and Mal A.K., "On the accuracy of approximate plate theories for wave field calculations in composite laminates,” Wave Motion, Vol. 21, 1995, pp. 17-34

10 Mal A.K. and Banerjee S., "Guided acoustic emission waves in a thick composite plate,” Proceedings of the SPIE, Vol. 5394, 2004, pp. 42-52

11 Moulin E., Assaad J., and Delebarre C., "Modeling of Lamb waves generated by integrated transducers in composite plates using a coupled finite element-normal modes expansion method," Journal of the Acoustical Society of America, Vol. 107, No. 1, January 2000, pp. 87-94

12 Lin X. and Yuan F. G., "Diagnostic Lamb waves in an integrated piezoelectric sensor/actuator plate: analytical and experimental studies,” Smart Materials and Structures, Vol. 10, 2001, pp. 907-913

13 Rose L.R.F. and Wang C.H., "Mindlin plate theory for damage detection: Source solutions," Journal of the Acoustical Society of America, Vol. 116, No. 1, 2004, pp. 154-171

14 Veidt M., Liu T. and Kitipornchai S., "Flexural waves transmitted by rectangular piezoceramic transducers,” Smart Materials and Structures, Vol. 10, 2001, pp. 681-688

15 Giurgiutiu V., "Lamb wave generation with piezoelectric wafer active sensors for structural health monitoring," Proceedings of the SPIE, Vol. 5056, 2003, pp. 111-122

16 Auld B.A., Acoustic fields and waves in solids Volumes I \& II, $2^{\text {nd }}$ ed., R.E. Kreiger Publishing Co., Florida, 1990

17 Crawley E.F. and de Luis J., “Use of piezoelectric actuators as elements of intelligent structures,” AIAA Journal, Vol. 25. No. 10, 1987, pp. 1373-1385

18 Miklowitz J., The theory of elastic waves and waveguides, North Holland, New York, USA, 1978

19 Graff K.F., Wave motion in elastic solids, Dover Publications, New York,1991

20 LAPACK user's guide $3^{\text {rd }}$ edition, Society for Industrial and Applied Mathematics, Philadelphia, 1999 